\title{
Unveiling Complex Plasmonic Resonances in Archimedean Nanospirals through Cathodoluminescence in a Scanning Transmission Electron Microscope
}

Jordan A. Hachtel ${ }^{1,2}$, Roderick B Davidson II ${ }^{1,3}$, Matthew F. Chisholm², Benjamin J. Lawrie ${ }^{3}$, Richard F. Haglund Jr. ${ }^{1}$, Sokrates T. Pantelides ${ }^{1,2}$

1. Department of Physics and Astronomy, Vanderbilt University, Nashville, TN, USA

2. Material Science and Technology Division, Oak Ridge National Laboratory, Oak Ridge, TN, USA

3. Quantum Information Science Group, Oak Ridge National Laboratory, Oak Ridge, TN, USA

Metallic nanostructures with a complex plasmonic response, such as the Archimedean nanospiral (ANS) present novel ways to utilize plasmonics in modern technology [1,2]. The nanospiral can support several resonant modes, with distinct electric field profiles as shown by finite-difference time-domain (FDTD) simulations such as the hourglass (500-650nm) and focusing $(650-980 \mathrm{~nm})$ modes [2]. In addition to the linear plasmonic response, the ANS exhibits a stronger second-order nonlinearity than seen in other metallic nanostructured systems. A high spatial-resolution picture of the plasmonic modes is critical to understanding the interactions between plasmonic modes that drive the high non-linear efficiencies [3].

Purely optical experiments struggle to observe the near field behavior of the structure due to being diffraction limited. However cathodoluminescence (CL) experiments in a scanning transmission electron microscope (STEM) create a unique opportunity for characterizing plasmonic systems with both the spatial sensitivity of electron optics and the spectral sensitivity of photonics. A significant benefit to the use of STEM-CL, as opposed to other high-resolution spectroscopy techniques such as electron energy loss spectroscopy (EELS), is that the photons generated from radiative decays in the sample are collected for signal instead of the electrons from the probe. As a result, the signal can be manipulated and filtered using standard optical techniques, allowing us to map difficult to observe plasmon modes and even give insight into the polarization of their radiative decay.

To experimentally observe the plasmon modes, an Au ANS array is fabricated using electron-beam lithography on a $50 \mathrm{~nm}$ silicon nitride film supported by a silicon substrate. A $500 \mu \mathrm{m} \times 500 \mu \mathrm{m}$ window is then backside-etched beneath the array. STEM-CL is done in a VG-HB601 STEM operated at $60 \mathrm{kV}$ with a home-built CL spectrometer system. Radiative emission is collected with a parabolic mirror and reflected out of a port in the side of the microscope, allowing the signal to be filtered and polarized using standard optical equipment, and ultimately collected in a photomultiplier tube (PMT).

First, the focusing mode (650-980nm) is examined. Fig 1a shows a FDTD simulation of the plasmon mode. Fig 1b shows a high angle annular dark field (HAADF) image of the fabricated ANS on the SiN window. The raw CL intensity is shown in Fig 1c with no spectral filtering. Emission from all plasmonic modes, interband transitions, and any other radiative decay pathways are all simultaneously detected. However, by spectrally filtering the CL signal, individual modes can be isolated. The focusing mode is predicted to be strongest in the $650-980 \mathrm{~nm}$ region, so by using a $600 \mathrm{~nm}$ long-pass spectral filter, the radiative decay from other optical features can be eliminated, and a map of the focusing mode (Figure 1d) can be obtained. For the hourglass mode, however, spectral filters cannot be used to observe the spatial profile of the plasmonic resonance, because unlike the focusing mode, the hourglass mode has a distinct polarization axis that is parallel to the axis of the exciting optical pulse, as shown in the FDTD simulation in Fig 1e. With no polarization selectivity on electron-optics, the hourglass plasmon modes 
can be excited in all in-plane axes of the ANS simultaneously within the STEM, and no individual plasmon can be isolated and detected. However, since the hourglass mode has a strong polarization dependence on the exciting pulse, it is likely that the resulting emission is similarly polarized. In Fig $1 \mathrm{f}$ the HAADF image of an ANS is shown, and Fig $1 \mathrm{~g}$ shows the unfiltered CL image. Fig $1 \mathrm{~h}$ shows CL image filtered with a linear polarizer and exhibits a similar spatial profile to the simulation in Fig 1e, demonstrating that the modes that have a have a strong dependence on the polarization of the exciting optical source also exhibit a polarization dependence on their radiative decay.

Nanostructures with complex, nonlinear plasmonic responses, such as the ANS, have unique optical attributes not present in simpler geometries. As a result, high spatial-resolution techniques to investigate the near-field profiles are an important avenue of plasmonic investigation. STEM-CL presents a particularly unique opportunity to combine photon and electron optics in order to characterize and map plasmonic modes, such as the hourglass and focusing modes of the ANS, with nanoscale precision.

\section{References:}

[1] AG Mark, JG Gibbs, TC Lee, et al., Nature Materials 12, (2013), p. 802-807

[2] JI Ziegler and RF Haglund, Plasmonics 8, (2012), p. 571-579

[3] RB Davidson, JI Ziegler, G Vargas, et al., Nanophotonics 4, (2015), p. 108-113

[4] This work was performed at Oak Ridge National Laboratory, operated by UT-Battelle for the U.S. Department of Energy under contract no. DE-AC05-00OR22725. J.A.H. and S.T.P. acknowledge funding from Department of Energy contract no. DE-FG02-09ER46554. All microscopy performed with support from the Department of Energy, Office of Science, Basic Energy Sciences, Materials Sciences and Engineering Division (J.A.H., S.T.P., M.F.C.) The nanofabrication was conducted at the Center for Nanophase Materials Sciences, which is a DOE Office of Science User Facility. The authors R.B.D. and B.J.L. recognize support from the Laboratory Directed Research and Development program.

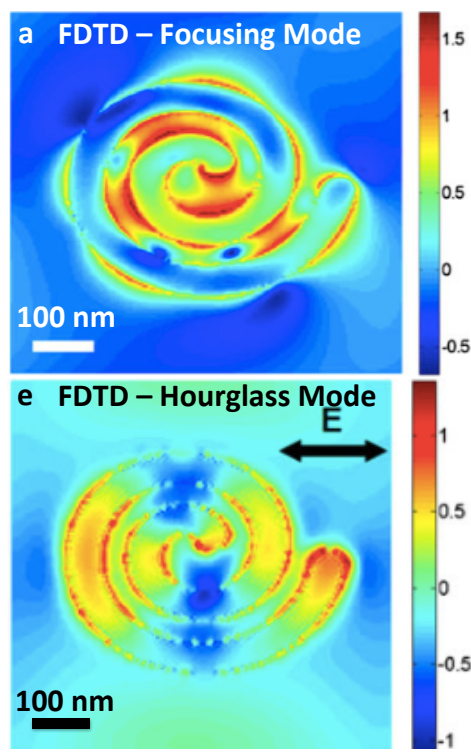

Figure 1. (a) Finite-difference time-domain (FDTD) simulation of the focusing mode in an Archimedean nanospiral (ANS). (b) High angle annular dark field (HAADF) image of an ANS and two cathodoluminescence (CL) images of the same ANS: (c) the unfiltered CL signal showing the total emission of the ANS, (d) the $600 \mathrm{~nm}$ long-pass filtered image showing the focusing mode. (e) FDTD simulation of the hourglass mode. (f) HAADF image of an ANS alongside (g) the unfiltered CL image and $(h)$ the CL image filtered with a linear polarizer. 